\title{
Homeobox-containing protein 1 loss is associated with clinicopathological performance in glioma
}

\author{
PING ZHANG ${ }^{1}$, QINGLIN LIU ${ }^{1}$, SHAOFENG YAN ${ }^{1-3}$, GUANG YUAN $^{1,4}$, JIE SHEN ${ }^{1}$ and GANG LI ${ }^{1}$ \\ ${ }^{1}$ Department of Neurosurgery, Qilu Hospital of Shandong University, Jinan, Shandong 250012; \\ ${ }^{2}$ Department of Neurosurgery, Beijing Tiantan Hospital, Capital Medical University; ${ }^{3}$ Beijing Key Laboratory of \\ Neurostimulation, Beijing 100050; ${ }^{4}$ Department of Neurosurgery, Central Hospital of Zibo, Zibo, Shandong 255000, P.R. China
}

Received March 13, 2016; Accepted February 24, 2017

DOI: $10.3892 / \mathrm{mmr} .2017 .7050$

\begin{abstract}
Homeobox-containing protein 1(HMBOX1) is a novel member of the homeobox family, and abnormal expression of HMBOX1 has been observed in several types of carcinoma. A total of 144 cases of confirmed glioma diagnoses were included in the present study. Grading was performed according to the World Health Organization (WHO) grading system for central nervous system neoplasm. Immunohistochemical staining of HMBOX1, proliferation marker protein Ki-67 (Ki-67) and microvessel density (MVD) was performed, and scores were calculated. HMBOX1 mRNA levels were detected using the reverse transcription quantitative polymerase chain reaction. It was identified that the expression of HMBOX1 was reduced in glioma tissue compared with normal brain tissue $(\mathrm{P}<0.05)$. The expression of HMBOX1 was downregulated significantly in WHO grade IV tumors compared with WHO grades II and III $(\mathrm{P}<0.05)$. HMBOX1 expression was significantly correlated with WHO grade, Karnofsky Performance Score, MVD and Ki-67 expression; however, not associated with age or gender. Log-rank testing did not demonstrate that HMBOX1 expression was associated with prognosis. In conclusion, HMBOX1 may be a potential diagnostic marker in glioma.
\end{abstract}

\section{Introduction}

Homeobox-containing protein 1 (HMBOX1) is a novel member of the homeobox family and was first identified from a pancreatic cDNA library in 2006 (1). It is expressed in the cytoplasm and nucleus of normal human cells, including cerebrum, pancreas, kidney and liver cells (2). HMBOX1 acts as a ubiquitous transcription repressor; it negatively regulates

Correspondence to: Dr Gang Li or Dr Jie Shen, Department of Neurosurgery, Qilu Hospital of Shandong University, 107 Wenhuaxi Road, Jinan, Shandong 250012, P.R. China

E-mail: ligangqiluhospital@163.com

E-mail: jackill2046@yeah.net

Key words: homeobox-containing protein 1, glioma, diagnostic marker natural killer (NK) cell function by suppressing the NKG2-D type II integral membrane protein/hematopoietic cell signal transducer signaling pathway (3). In addition, HMBOX1 is a key factor in the differentiation of bone marrow stromal cells into vascular endothelial cells (4). Abnormal expression of HMBOX1 has been observed in several types of carcinoma, suggesting that it may be involved in the pathobiology of tumors. HMBOX1 is more highly expressed in clear-cell carcinoma originating from the renal tubule; however, expressed at markedly deceased levels in liver cancer compared with the adjacent normal tissues (2). At present, the expression and function of HMBOX1 remains to be investigated in human glioma.

Glioma is the most common type of malignant tumor in the central nervous system, with an incidence of 5 cases/100,000 persons worldwide annually (5). Glioma is currently diagnosed based on histomorphologic criteria and graded between I and IV, according to the World Health Organization (WHO) classification system for primary brain tumors (6). The algorithm of diagnosis and classification helps clinicians to estimate the prognosis of the natural course of the disease. The median survival time for patients of grade I/II glioma is between 4.7 and 9.8 years (7). However, the average survival time remains poor for individuals with grade III/IV glioma (5), at 12.1 months with concurrent radiation and temozolomide therapy (8). At present, age and patient performance scale score (Karnofsky Performance Score; KPS) are regarded as the overall accepted prognostic factors (9). Current research efforts are focused on understanding the molecular events of glioma, in order to identify effective prognostic and predictive markers, as well as targets for the development of more effective chemotherapeutic drugs.

Understanding the tumor biology of glioma has diagnostic importance and provides useful prognostic information. In the present study, HMBOX1 expression in glioma of differing WHO grades was investigated, and a correlative analysis performed of its expression compared with clinical pathologic factors and the prognosis of glioma patients. It was observed that HMBOX1 was downregulated in glioma and exhibited a significant correlation with WHO grade, KPS, microvessel density (MVD) and proliferation marker protein Ki-67 (Ki-67) expression, indicating that HMBOX1 may serve an important role in gliomagenesis. 


\section{Materials and methods}

Human samples. The present study was approved by the Research Ethics Committee of Qilu Hospital, Shandong University (Jinan, China). Informed consent was obtained from all patients included in the study. The histological and clinical diagnoses of the tumors in all these patients were performed by the Department of Pathology, Qilu Hospital.

A total of 57 new glioma samples were collected from the neurosurgery department of Qilu Hospital between October 2012 and May 2013 according to the National Regulation of Clinical Sampling in China $(10,11)$. Tumors were removed with wide tumor-free resection margins of $1-2 \mathrm{~cm}$. A large amount of peritumoral tissue/cortex (macroscopically normal-appearing cortex/white matter adjacent to the mass lesion; however, microscopically containing infiltrating tumor cells in astrocytoma and glioblastoma cases) was also resected. The adult control brain samples were collected through surgical resection in nine trauma patients. One section of the tissue was immediately fixed in $10 \%$ neutral buffered formalin for histological analysis and the remainder was immediately frozen in liquid nitrogen for reverse transcription quantitative polymerase chain reaction (RT-qPCR) analysis, and conserved until use.

Additionally, 17 paraffin-embedded pathological specimens of glioma were obtained from the archives of the Department of Pathology, Qilu Hospital, between October 2012 and May 2013. The cases were selected based on the following criteria: Pathological diagnosis of glioma; primary and curative resection of tumor without preoperative or postoperative anticancer treatment; and the availability of resected tissue and follow-up data.

Glioma tissue microarrays (TMAs) were obtained from Cybrdi (Xi'an, China). The array dot diameter was $1.5 \mathrm{~mm}$, and each dot represented a tissue spot from one individual specimen that was selected. All 70 glioma samples (42 females and 28 males, aged between 2 and 72) were verified by pathological analysis and classified according to the WHO classification standard. Patient characteristics are displayed in Table I.

All patients were followed up for 3 years, or until mortality. Overall survival was calculated from the date of the initial surgery to the date of mortality. Patients who succumbed to diseases not directly associated with their glioma, or due to unexpected events, were excluded from the present study.

Immunohistochemistry (IHC). The IHC experiment was performed using the strepto-avidin-biotin complex method. The primary antibodies used were as follows: Rabbit anti-HMBOX1 antibody (cat. no. SAB2700278; 1:1,000 Sigma-Aldrich; Merck KGaA, Darmstadt, Germany); rabbit cluster of differentiation (CD) 34 (BI-3c5; cat. no. sc-19621; 1:100; Santa Cruz Biotechnology, Inc., Dallas, TX, USA); and mouse Ki-67 (DAKO; cat. no. M7240; 1:150; Agilent Technologies, Inc., Santa Clara, CA, USA). In the negative control, the primary antibodies were replaced with PBS. Tumor-containing sections and TMAs were heated at $60^{\circ} \mathrm{C}$ for $30 \mathrm{~min}$, deparaffinized in xylene, and rehydrated in graded concentrations of ethanol. Heat-induced antigen retrieval [10 $\mathrm{mM}$ citrate buffer $\left(\mathrm{pH} 6.0\right.$ ) at $96^{\circ} \mathrm{C}$ for $15 \mathrm{~min}$ in a thermostat-controlled water bath] was used. Endogenous peroxidase activity was blocked by incubation in $0.3 \%$ hydrogen peroxide at $37^{\circ} \mathrm{C}$ for $15 \mathrm{~min}$. Nonspecific binding of primary antibodies was blocked by normal serum (cat. no. SAP-9100OriGene Technologies, Inc., Beijing, China) from the same species as that of secondary antibodies at $37^{\circ} \mathrm{C}$ for $25 \mathrm{~min}$. Immunostaining involved sequential applications of primary antibody at $4^{\circ} \mathrm{C}$ overnight, followed by biotinylated secondary antibodies (cat. no. SAP-9100; OriGene Technologies, Inc.) at $37^{\circ} \mathrm{C}$ for $30 \mathrm{~min}$. Samples were subsequently reacted with diaminobenzidine and counterstained. Mayer's hematoxylin was used as a nuclear counterstain, except in Ki-67 immunostaining in which eosin was used as a cytoplasmic counterstain.

Sections were examined and scored for immunoreactivity for anti-HMBOX1 antibody. The percentages and intensity of positive staining in 10 high-power randomly-selected fields were evaluated by two investigators in a blinded manner. The percentages of positively stained tumor cells in a field were scored as follows: 0 , none; $1,<10 \% ; 2,10-50 \%$; and 3, $>50 \%$. The staining intensity in a field was scored as follows: 0 , no staining; 1 , weak staining appearing as light yellow in color; 2 , moderate staining appearing as yellowish-brown in color; and 3 , strong staining appearing as brown in color. The immunoreactivity product (IRP) of individual sections was calculated as: Averaged staining intensity score $\mathrm{x}$ proportion score. Scores for Ki-67 were recorded as the number of immunopositive cells/high-power fields. MVD was counted as the CD34 positive vessels or clusters of cells/high-power fields. The number of immunopositive cells or vessels under 5 fields/slide with the highest counts was counted and the average was recorded.

Immunofluorescence labeling. Glioma cell lines $\mathrm{T} 98 \mathrm{G}$ and U251were obtained from American Type Culture Collection (Manassas, VA, USA) and conserved in our laboratory for $<2$ years. Cells were cultured in Dulbecco's modified Eagle's medium (DMEM; Hyclone; GE Healthcare Life Sciences, Logan, UT, USA) supplemented with $10 \%$ fetal bovine serum (FBS; Gibco; Thermo Fisher Scientific, Inc., Waltham, MA, USA), penicillin $(100 \mathrm{U} / \mathrm{ml})$, and streptomycin $(100 \mu \mathrm{g} / \mathrm{ml})$. NK-92 were obtained from the Institute of Immunopharmacology and Immunotherapy, School of Pharmaceutical Sciences (Shandong University) and cultured in Minimum Essential Medium Alpha (Gibco, Thermo Fisher Scientific, Inc.) containing $12.5 \%$ horse serum (Gibco, Thermo Fisher Scientific, Inc.), 12.5\% FBS (Sijiqing, Hangzhou, China), $100 \mathrm{U} / \mathrm{ml}$ recombinant human interleukin-2 (Changsheng, Changchun, China), and $0.1 \mathrm{mM} \beta$-mercaptoethanol. All cells were maintained at $37^{\circ} \mathrm{C}$ in a humidified incubator with $5 \%$ $\mathrm{CO}_{2}$. Cells were plated on coverslips $\left(5 \times 10^{4}\right.$ cells/well) coated with poly-L-lysine. Cells were fixed in $4 \%$ paraformaldehyde and permeabilized with $0.1 \%$ Triton X-100 (cat. no. T8787; Sigma-Aldrich; Merck KGaA). Subsequently, cells were incubated with primary antibody: Anti-HMBOX1 $(1: 1,000)$ overnight at $4^{\circ} \mathrm{C}$. Tetramethylrhodamine-conjugated anti-rabbit IgG (cat. no. sc-2492; 1:100; Santa Cruz Biotechnology, Inc.) was used as the secondary antibody. The cells were incubated with secondary antibody for $1 \mathrm{~h}$ at $37^{\circ} \mathrm{C}$. Coverslips were mounted in $90 \%$ glycerol/PBS and analyzed using an Olympus BX61 fluorescence microscope (Olympus Corporation, Tokyo, Japan).

$R T$ - $q P C R$. The total RNA from the freshly-frozen samples was extracted using Trizol reagent (Invitrogen; Thermo Fisher 
Table I. Expression of HMBOX1, Ki-67, MVD and other clinicopathological factors in glioma of different WHO classifications.

\begin{tabular}{lccccc}
\hline Tissue & Gender (M:F) & Mean age (range) & Mean MVD (range) & Mean Ki-67 (range) & Mean HMBOX1 (range) \\
\hline Normal & $5: 4$ & $46.4(5-71)$ & $26.6(11.6-45.8)$ & $2.4(1.7-2.9)$ & $8.1(6-9)$ \\
WHO II & $30: 28$ & $40.9(14-65)$ & $29.2(14.2-46.2)$ & $3.3(2.4-4.7)$ & $5.3(1-9)$ \\
WHO III & $23: 10$ & $47.8(9-72)$ & $41.7(26.2-56.0)$ & $11.8(9.1-16.6)$ & $6.0(1-9)$ \\
WHO IV & $26: 27$ & $42.3(12-71)$ & $48.1(36.6-64.8)$ & $24.2(7.2-45.6)$ & $2.8(0-6)$ \\
\hline
\end{tabular}

HMBOX1, homeobox-containing protein 1; Ki-67, proliferation marker protein Ki-67; MVD, microvessel density; WHO, World Health Organization.

Scientific, Inc.). A total of $500 \mathrm{ng}$ RNA was transcribed into first strand cDNA using the primescript ${ }^{\mathrm{TM}} \mathrm{RT}$ reagent kit (Toyobo, Tokyo, Japan). The qPCR was performed in a total volume of $20 \mu \mathrm{l}$, according to the manufacturer's protocol of SYBR PremixEx Taq ${ }^{\mathrm{TM}}$ (Takara Bio, Inc., Otsu, Japan). The sequences of primers were: HMBOX1 forward, 5'-GATGAT GTCGACGGGAATGACTAC-3' and reverse, 5'-CTGCTG CCATTTCCACAGCTAA-3'; and GAPDH forward, 5'-CAA CAGCGACACCCACTCCT-3' and reverse, 5'-CACCCTGTT GC-TGTAGCCAAA-3'. The amplification was performed using a Lightcycler 2.0 instrument (Roche Applied Science, Penzburg, Germany). Cycling conditions were as follows: An initial predenaturation at $95^{\circ} \mathrm{C}$ for $30 \mathrm{sec}$, followed by 40 cycles of denaturation at $95^{\circ} \mathrm{C}$ for $5 \mathrm{sec}$, annealing at $60^{\circ} \mathrm{C}$ for $20 \mathrm{sec}$ and extension at $72^{\circ} \mathrm{C}$ for $5 \mathrm{~min}$. Gene expression levels of HMBOX1 was determined by the mRNA level quantified by the calibration curve of the standards and normalized to the level of the reference gene, GAPDH (12).

Statistical analysis. Data are presented as mean \pm standard deviation. The comparisons between two groups were completed using a Mann-Whitney test. The Kruskal-Wallis test was employed to calculate all grouped P-values $(n \geq 3)$. Spearman's rank correlation analysis was used in the analysis of the correlation among HMBOX1 and KPS, WHO grade, MVD, Ki-67, age and gender. The overall survival of the different groups of patients was estimated using the Kaplan-Meier survival curve and analyzed using log-rank tests. All statistical analyses were performed using SPSS software (version 11.0; SPSS, Inc., Chicago, IL, USA). P<0.05 was considered to indicated a statistically significant difference.

\section{Results}

HMBOX1 protein and $m R N A$ expression in human glioma tissues. IHC staining of HMBOX1 in paraffin-embedded glioma tissues and TMAs was performed. In accordance with previous results (2), HMBOX1 was expressed in the nucleus and cytoplasm of normal and tumor tissues (Fig. 1). The expression of HMBOX1 was further validated by immunofluorescence to be localized within the nucleus and cytoplasm in the human glioma cell lines T98G and U251. The NK cell line NK-92 was used as a positive control (Fig. 2).

It was observed that HMBOX1 was expressed in $100 \%$ (9/9) of the normal tissue samples and 92\% (132/144) of the glioma samples, including all collected samples and the TMA.
A moderate or low level of expression of HMBOX1 was observed in a number of glioma tissues (Table I), whereas the HMBOX1 expression was high in the majority of the normal tissues. The decreased expression of HMBOX1 in glioma was confirmed by IHC using commercially available TMAs, and was able to differentiate between normal and glioma tissue $(\mathrm{P}<0.01$; Fig. 3A). In addition, HMBOX1 expression was significantly decreased in WHO grade IV compared with WHO grade II and III glioma $(\mathrm{P}<0.05$; Fig. $3 \mathrm{~B})$, which was confirmed by RT-qPCR (P<0.05; Fig. 3C).

Association of HMBOX1 with clinicopathological parameters of glioma. As the expression of HMBOX1 was significantly reduced in glioma, particularly in WHO grade IV, the association between HMBOX1 expression and the clinicopathological features of glioma patients was analyzed. It was demonstrated that HMBOX1 demonstrated a significant correlation with WHO grades $(r=-0.8423 ; \mathrm{P}<0.001)$ and KPS $(r=-0.4924$; $\mathrm{P}<0.001)$. A significant correlation between HMBOX1 and MVD expression was identified $(\mathrm{r}=-0.526 ; \mathrm{P}=0.017)$, suggesting that HMBOX1 is involved in blood vessel generation in glioma. HMBOX1 expression significantly correlates with Ki-67 $(r=-0.479$; $\mathrm{P}=0.044)$; however, not with age or gender (Table II).

Association of HMBOX1 expression with overall survival in patients with glioma. HMBOX1 expression in high-grade glioma (WHO grade IV) was decreased compared with low-level (WHO grades II and III) glioma, and its expression was associated with WHO grade and KPS suggesting that the expression level of HMBOX1 may be associated with prognosis or recurrence. The patients were followed up for 8-36 months; the median follow-up time was 29 months. However, only 41/74 cases were available and 26/41 patients (63.4\%) had succumbed to the disease. Kaplan-Meier analysis and log-rank testing was used to assess the correlation between HMBOX1 expression and the prognosis of glioma patients. The median survival time of patients with low HMBOX1 expression [immunoreactive product (IRP) <3] was 18.7 months; the median survival time of the high expression group (IRP >3) was 28.3 months. The survival rate of patients between these two groups was not significantly different according to the log-rank test (data not shown).

\section{Discussion}

To the best of our knowledge, the present study is the first to investigate the correlation between HMBOX1 expression 


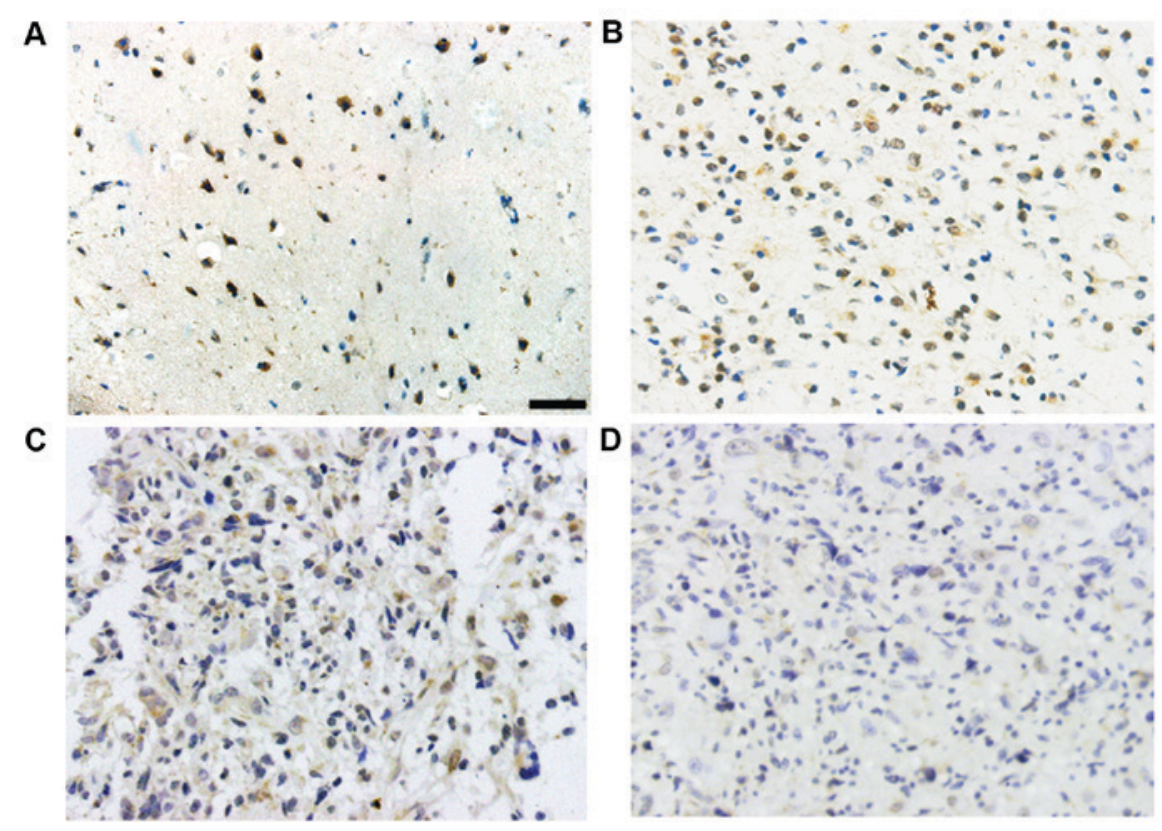

Figure 1. HMBOX1 expression levels in the normal brain and glioma samples. Immunohistochemical staining of HMBOX1 in (A) normal brain samples, (B) WHO grade II glioma, (C) WHO grade III glioma, (D) WHO grade IV glioma (magnification, x200). Scale bar, $25 \mu$ m. HMBOX1, homeobox-containing protein 1; WHO, World Health Organization.
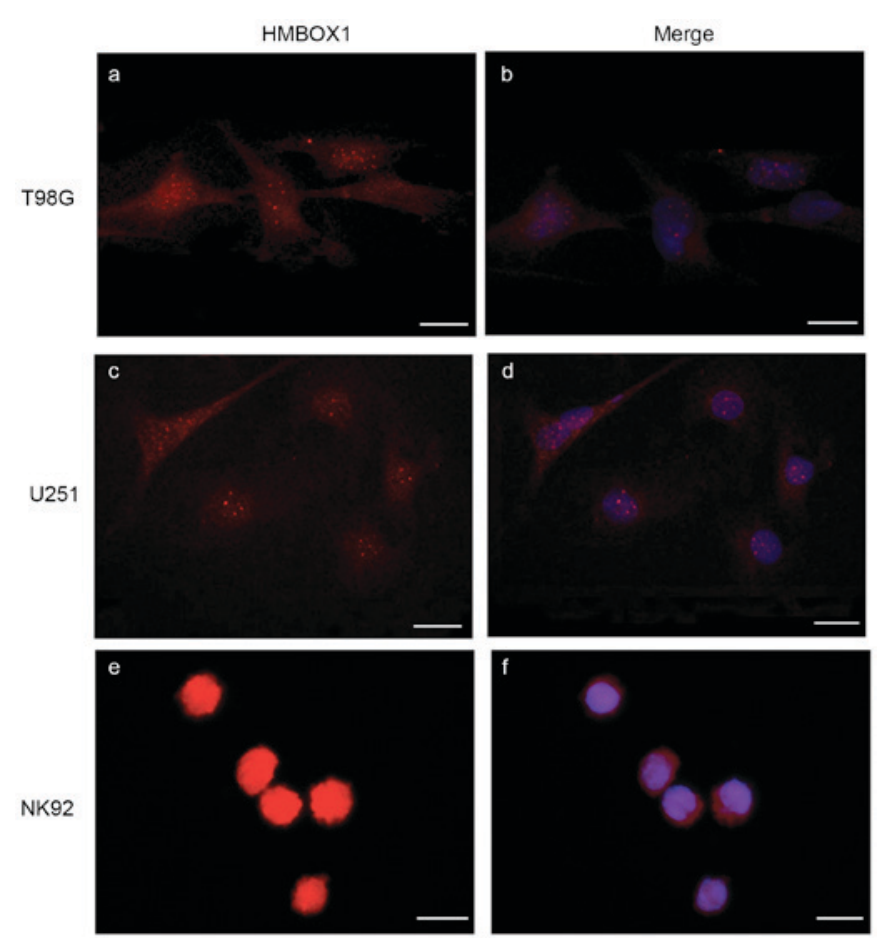

Figure 2. Expression of HMBOX1 is localized in the nucleus and cytoplasm in the human glioma cell lines T98 and U251, using immunofluorescence. The natural killer cell line NK-92 was used as a positive control. Cells were grown on cover glass, fixed, and stained for HMBOX1 (red) and nucleus (blue). Magnification, x400. Scale bar, $12.5 \mu \mathrm{m}$. (a) HMBOX1 and (b) merged staining for T98 cells; (c) HMBOX1 and (d) merged staining for U251 cells; and (e) HMBOX1 and (f) merged staining for NK-92 cells. HMBOX1, homeobox-containing protein 1 .

and glioma. The present study revealed that HMBOX1 was widely expressed in the cytoplasm and nuclei of normal brain and glioma tissues. HMBOX1 expression levels were distinct between normal brain and glioma tissues. A significant reduction in HMBOX1 expression was observed in WHO grade IV compared with WHO grades II and III. HMBOX1 expression was significantly correlated with WHO grade, KPS, MVD and Ki-67 expression; however, not associated with age or gender.

HMBOX1 is a ubiquitous transcription repressor in human tissues $(1-3,13,14)$. Aberrant expression of transcription repressors has been reported in numerous types of tumor, including leukemia, breast, and prostate cancer (15). Reduced expression of a repressor may lead to hyper-activation of tumor-promoting genes (16). HMBOX1 expression was reduced in glioma compared with normal brain tissue, which was previously reported in hepatocellular carcinoma (2). This observation suggests that HMBOX1 has potential diagnostic value in distinguishing glioma from normal brain tissues.

The expression level of HMBOX1 is associated with the WHO classification. In particular, compared with WHO grades II and III, the expression of HMBOX1 was reduced significantly in WHO grade IV glioma. These results suggest that HMBOX1 may suppress malignancy and metastasis of tumor cells.

KPS was designed for cancer patients to measure the level of patient activity and medical requirements (17) The benefit of therapeutic interventions has been questioned for patients with a KPS score $\leq 50$ (18). KPS has been demonstrated to be a strong, independent predictor of survival, and a low KPS score is associated with reduced median survival (19). The correlation between HMBOX1 and KPS score indicates that HMBOX1 may serve an important role in therapeutic decision-making and prognosis.

Tumor angiogenesis, the formation of new blood vessels from pre-existing blood vessels, is essential for tumor growth $(20,21)$. MVD is used to assess the angiogenesis in breast, stomach, bladder and prostate cancer, and brain glioma (22-25). Glioma vessels are structurally and 
Table II. Correlational analysis of HMBOX1 expression with MVD, Ki-67, age and gender.

\begin{tabular}{lrc}
\hline & \multicolumn{2}{c}{ HMBOX1 } \\
\cline { 2 - 3 } Clinicopathological factors & $\mathrm{r}^{\mathrm{a}}$ & P-value \\
\hline WHO grade & -0.842 & $<0.001$ \\
KPS & 0.492 & $<0.001$ \\
MVD & -0.536 & 0.017 \\
Ki-67 & -0.286 & 0.04 \\
Age & 0.395 & 0.077 \\
Gender & 0.099 & 0.256 \\
\hline
\end{tabular}

${ }^{\mathrm{a}} \mathrm{r}$ is considered to be significant when $\mathrm{P}<0.05$. HMBOX1, homeobox-containing protein 1 ; Ki-67, proliferation marker protein Ki-67; MVD, microvessel density; KPS, Karnofsky Performance Score; WHO, World Health Organization.

functionally distinct from normal vessels (26). The endothelial-lined vasculature connects with vasculogenic mimicry channels, which are non-endothelial, tumor-cell-lined microvascular channels (27). The present study demonstrates that HMBOX1 expression and MVD in glioma were significantly negatively-associated. HMBOX1 is regarded as a key factor in the differentiation of bone marrow stromal cells into functional vascular endothelial cells (28), therefore the downregulation of HMBOX1 may contribute to aberrant angiogenesis in glioma.

$\mathrm{Ki}-67$ is a proliferative marker and is associated with poor prognosis in glioma, ependymoma and pituitary adenoma (29-35). The present study revealed that HMBOX1 expression was significantly negatively-associated with Ki-67 in glioma $(\mathrm{P}<0.05)$, implying that HMBOX1 has a negative role in gliomagenesis.

Although the clinical indicators, including WHO grade and stage, as well as KPS and age have been demonstrated to exhibit prognostic ability, few molecular markers are used in clinical practice for glioma prognosis. Numerous biomarkers have been identified, including epidermal growth factor receptor, protein phosphatase and tensin homolog on chromosome 10, and O6-methylguanine-DNA methyltransferase, which were demonstrated to be potentially important prognostic and predictive indicators of glioma (36). The present study also investigated the potential ability of HMBOX1 as a prognostic indicator of glioma. Log-rank testing indicated that the survival and relapse rates of patients in the high HMBOX1 expression group compared with the low expression group were not significantly different. However, the mean survival time of the low expression group was decreased compared with that of the high expression group. A limitation of the present study is that only $41 / 74$ cases were able to be followed up. The prognostic ability of HMBOX1 requires further research.

In conclusion, HMBOX1 is differentially expressed in glioma compared with normal brain tissue. A significant reduction of HMBOX1 expression was observed in WHO grade IV glioma compared with WHO grades II and III. HMBOX1 expression and WHO grade, KPS, tumor angiogenesis and
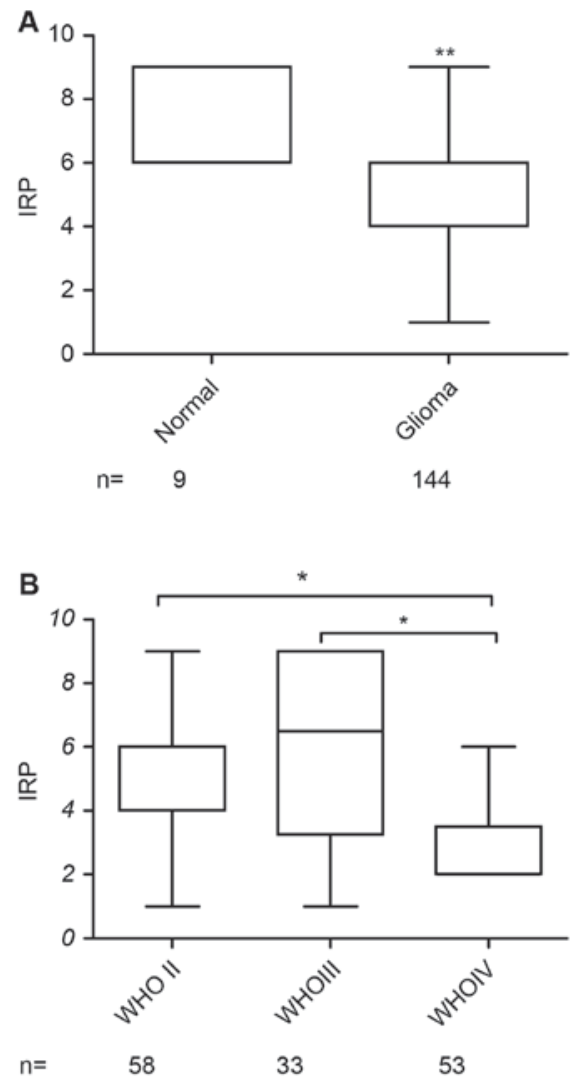

$\begin{array}{llll}n= & 58 & 33 & 53\end{array}$

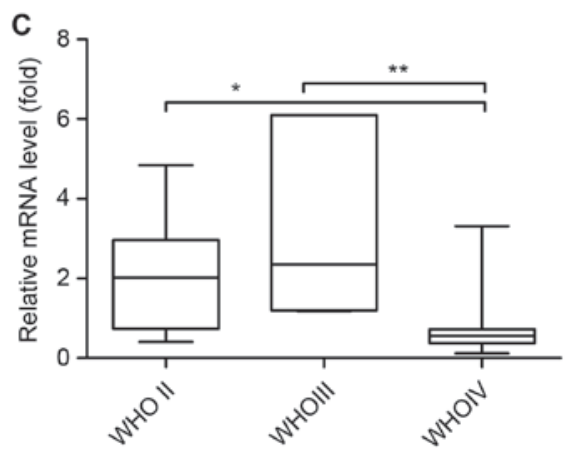

$\mathrm{n}=\quad 21 \quad 16 \quad 20$

Figure 3. HMBOX1 is able to distinguish glioma from normal brain tissue. (A) HMBOX1 immunohistochemistry demonstrates that the expression of HMBOX1 in glioma was significantly decreased compared with the normal brain tissue. (B) Data from the HMBOX1 immunohistochemistry was stratified by WHO grade (II-IV). P-values were calculated using a Kruskal-Wallis test. (C) Data from HMBOX1 polymerase chain reaction analysis was stratified by WHO grade (II-IV). P-values were calculated using a Kruskal-Wallis test. ${ }^{*} \mathrm{P}<0.05 ;{ }^{* *} \mathrm{P}<0.01$. HMBOX1, homeobox-containing protein 1 ; IRP, immunoreactivity product; WHO, World Health Organization.

proliferation are significantly correlated. The expression of HMBOX1 is not associated with prognosis. However, the role and mechanisms of HMBOX1 in glioma require further investigation.

The present results are based on a single hospital-based retrospective study. It should be noted that there may be unmeasured differences that may distort the results. A community-based or multicenter prospective study, including more potential confounds, is required. Based on the above results, HMBOX1 may be a potential diagnostic marker in glioma. 


\section{Acknowledgements}

The present study was supported by the Shandong Provincial Science and Technology Program (grant no. BS2010SW013) and the Taishan Scholars of Shandong Province of China (grant no. ts201511093).

\section{References}

1. Chen S, Saiyin H, Zeng X, Xi J, Liu X, Li X and Yu L: Isolation and functional analysis of human HMBOX1, a homeobox containing protein with transcriptional repressor activity. Cytogenet Genome Res 114: 131-136, 2006.

2. Dai J, Zhang C, Tian Z and Zhang J: Expression profile of HMBOX1, a novel transcription factor, in human cancers using highly specific monoclonal antibodies. Exp Ther Med 2: 487-490, 2011.

3. Wu L, Zhang $\mathrm{C}$ and Zhang J: HMBOX1 negatively regulates NK cell functions by suppressing the NKG2D/DAP10 signaling pathway. Cell Mol Immunol 8: 433-440, 2011.

4. Han L, Shao J, Su L, Gao J, Wang S, Zhang Y, Zhang S, Zhao B and Miao J: A chemical small molecule induces mouse embryonic stem cell differentiation into functional vascular endothelial cells via Hmbox1. Stem Cells Dev 21: 2762-2769, 2012.

5. Wen PY and Kesari S: Malignant gliomas in adults. N Engl J Med 359: 492-507, 2008

6. Tabatabai G, Hegi M, Stupp R and Weller M: Clinical implications of molecular neuropathology and biomarkers for malignant glioma. Curr Neurol Neurosci Rep 12: 302-307, 2012.

7. Kesari S, Schiff D, Drappatz J, LaFrankie D, Doherty L, Macklin EA, Muzikansky A, Santagata S, Ligon KL, Norden AD, et al: Phase II study of protracted daily temozolomide for low-grade gliomas in adults. Clin Cancer Res 15: 330-337, 2009.

8. Stupp R, Mason WP, van den Bent MJ, Weller M, Fisher B, Taphoorn MJ, Belanger K, Brandes AA, Marosi C, Bogdahn U, et al: Radiotherapy plus concomitant and adjuvant temozolomide for glioblastoma. N Engl J Med 352: 987-996, 2005.

9. Stark AM, Stepper W and Mehdorn HM: Outcome evaluation in glioblastoma patients using different ranking scores: KPS, GOS, mRS and MRC. Eur J Cancer Care (Engl) 19: 39-44, 2010.

10. Zhang Y, Hao H, Zhao S, Liu Q, Yuan Q, Ni S, Wang F, Liu S, Wang L and Hao A: Downregulation of GRIM-19 promotes growth and migration of human glioma cells. Cancer Sci 102: 1991-1999, 2011.

11. Niu CS, Yang Y and Cheng CD: MiR-134 regulates the proliferation and invasion of glioblastoma cells by reducing Nanog expression. Int J Oncol 42: 1533-1540, 2013.

12. Hou X, Chen X, Zhang P, Fan Y, Ma A, Pang T, Song Z, Jin Y, Hao W, Liu F, et al: Inhibition of hedgehog signaling by GANT58 induces apoptosis and shows synergistic antitumor activity with AKT inhibitor in acute T cell leukemia cells. Biochimie 101: 50-59, 2014.

13. Gong J, Liu R, Zhuang R, Zhang Y, Fang L, Xu Z, Jin L, Wang T, Song $\mathrm{C}$, Yang $\mathrm{K}$, et al: miR-30c-1* promotes natural killer cell cytotoxicity against human hepatoma cells by targeting the transcription factor HMBOX1. Cancer Sci 103: 645-652, 2012.

14. Dai J, Wu L, Zhang C, Zheng X, Tian Z and Zhang J: Recombinant expression of a novel human transcriptional repressor HMBOX1 and preparation of anti-HMBOX1 monoclonal antibody. Cell Mol Immunol 6: 261-268, 2009.

15. Vaiopoulos AG, Kostakis ID, Athanasoula $\mathrm{KCh}$ and Papavassiliou AG: Targeting transcription factor corepressors in tumor cells. Cell Mol Life Sci 69: 1745-1753, 2012.

16. Hurst DR and Welch DR: Unraveling the enigmatic complexities of BRMS1-mediated metastasis suppression. FEBS Lett 585: $3185-3190,2011$
17. Ellison RR, Karnofsky DA and Burchenal JH: Clinical evaluation of 6-chloropurine in leukemia of adults. Blood 13: 705-724, 1958.

18. Marina O, Suh JH, Reddy CA, Barnett GH, Vogelbaum MA, Peereboom DM, Stevens GH, Elinzano $H$ and Chao ST: Treatment outcomes for patients with glioblastoma multiforme and a low Karnofsky Performance Scale score on presentation to a tertiary care institution. Clinical article. J Neurosurg 115: 220-229, 2011.

19. Patil CG, Yi A, Elramsisy A, Hu J, Mukherjee D, Irvin DK, Yu JS, Bannykh SI, Black KL and Nuño M: Prognosis of patients with multifocal glioblastoma: A case-control study. J Neurosurg 117: 705-711, 2012.

20. Hanahan D and Weinberg RA: Hallmarks of cancer: The next generation. Cell 144: 646-674, 2011.

21. Carmeliet P and Jain RK: Molecular mechanisms and clinical applications of angiogenesis. Nature 473: 298-307, 2011.

22. Leon SP, Folkerth RD and Black PM: Microvessel density is a prognostic indicator for patients with astroglial brain tumors. Cancer 77: 362-372, 1996.

23. Weidner N, Semple JP, Welch WR and Folkman J: Tumor angiogenesis and metastasis-correlation in invasive breast carcinoma. N Engl J Med 324: 1-8, 1991.

24. Weidner N, Carroll PR, Flax J, Blumenfeld W and Folkman J: Tumor angiogenesis correlates with metastasis in invasive prostate carcinoma. Am J Pathol 143: 401-409, 1993.

25. Bochner BH, Cote RJ, Weidner N, Groshen S, Chen SC, Skinner DG and Nichols PW: Angiogenesis in bladder cancer: Relationship between microvessel density and tumor prognosis. J Natl Cancer Inst 87: 1603-1612, 1995.

26. Stiver SI: Angiogenesis and its role in the behavior of astrocytic brain tumors. Front Biosci 9: 3105-3123, 2004.

27. Chen YS and Chen ZP: Vasculogenic mimicry: A novel target for glioma therapy. Chin J Cancer 33: 74-79, 2014.

28. Su L, Zhao H, Sun C, Zhao B, Zhao J, Zhang S, Su H and Miao J: Role of Hmbox1 in endothelial differentiation of bone-marrow stromal cells by a small molecule. ACS Chem Biol 5: 1035-1043, 2010.

29. Kontogeorgos G: Predictive markers of pituitary adenoma behavior. Neuroendocrinology 83: 179-188, 2006.

30. Gejman R, Swearingen B and Hedley-Whyte ET: Role of Ki-67 proliferation index and p53 expression in predicting progression of pituitary adenomas. Hum Pathol 39: 758-766, 2008.

31. Bruna J, Brell M, Ferrer I, Gimenez-Bonafe P and Tortosa A: Ki-67 proliferative index predicts clinical outcome in patients with atypical or anaplastic meningioma. Neuropathology 27: 114-120, 2007.

32. Preusser M, Heinzl H, Gelpi E, Höftberger R, Fischer I, Pipp I, Milenkovic I, Wöhrer A, Popovici F, Wolfsberger S and Hainfellner JA: Ki67 index in intracranial ependymoma: A promising histopathological candidate biomarker. Histopathology 53: 39-47, 2008.

33. Wolfsberger S, Fischer I, Höftberger R, Birner P, Slave I, Dieckmann K, Czech T, Budka H and Hainfellner J: Ki-67 immunolabeling index is an accurate predictor of outcome in patients with intracranial ependymoma. Am J Surg Pathol 28: 914-920, 2004.

34. Rodriguez-Pereira C, Suárez-Peñaranda JM, Vázquez-Salvado M, Sobrido MJ, Abraldes M, Barros F and Forteza J: Value of MIB-1 labelling index (LI) in gliomas and its correlation with other prognostic factors. A clinicopathologic study. J Neurosurg Sci 44: 203-210, 2000.

35. Heegaard S, Sommer HM, Broholm H and Broendstrup O: Proliferating cell nuclear antigen and Ki-67 immunohistochemistry of oligodendrogliomas with special reference to prognosis. Cancer 76: 1809-1813, 1995.

36. Ma R, de Pennington N, Hofer M, Blesing C and Stacey R: Diagnostic and prognostic markers in gliomas- an update. $\mathrm{Br} \mathrm{J}$ Neurosurg 27: 311-315, 2013. 